\title{
Superinfecção e rotura de aneurisma da aorta abdominal por Salmonella dublin: relato de caso
}

\author{
Anibal BASILE FILHO*, Albert JAEGER ${ }^{\star *}$, Antônio CAPONE NETO*, Mário MANTOVANI*
}

RBCCV $44205-71$

BASILE FILHO, A.; JAEGER, A.; CAPONE NETO, A.; MANTOVANI, M. - Superinfecção e rotura de aneurisma da aorta abdominal por Salmonella dublin: relato de caso. Rev. Bras. Cir. Cardiovasc., 3(3): 220-223, 1988.

RESUMO: Os autores relatam o caso de paciente de 66 anos, que apresentou superinfecçāo e rotura de aneurisma da aorta abdominal, após septicemia por Salmonella dublin. As infecçōes endovasculares associadas à rotura de aneurisma pré-existentes são um rico potencial nos pacientes com mais de 50 anos de idade, que apresentam bacteremia ou septicemia por Salmonella sp. A alta mortalidade da aortite por salmonelose é devida à septicemia grave ou à rotura desses aneurismas. Qualquer tecido orgânico pode ser a sede de infecçōes metastáticas, porém os locais mais susceptiveis são os tecidos necróticos e as lesōes crônicas degenerativas. Os autores discutem a importância do diagnóstico precoce para reduzir a mortalidade dessa entidade.

DESCRITORES: aneurisma de aorta abdominal, rotura, Salmonella dublin; aneurismas de aorta abdominal, cirurgia.

\section{INTRODUÇĀO}

As bacteremias causadas por Salmonella $s p$ são devidas a um número restrito de espécies, particularmente Salmonella typhi-murium, Salmonella choleraesuis, Salmonela enteritis e, mais recentemente, Salmonella dublin ${ }^{3}$.

Qualquer tecido orgânico pode ser sediado pela Salmonella, durante uma bacteremia, ou septicemia. Contudo, parece haver uma preferência por tecidos necróticos e as lesōes degenerativas crônicas. Assim, essas infecçōes metastáticas podem desenvolver-se em hematomas, tumores malignos, cistos ou no tecido endovascular alterado, como, por exemplo, a aterosclerose ${ }^{6}$.

A infecção secundária à salmonelose não é muito freqüente, porém, deve ser sempre lembrada, diante da persistência de sinais infecciosos em um paciente com antecedentes de aneurisma arterial e idade superior a 50 anos $^{4}$. Nesse caso, a superinfecção do aneurisma conduz, freqüentemente, à sua rotura ${ }^{3,4,14}$.

O caso relatado é de paciente com antecedentes de aterosclerose e aneurisma da aorta abdominal, que apresentou septicemia por Salmonella dublin e rotura do aneurisma.

\section{RELATO DO CASO}

Paciente do sexo feminino, com 66 anos de idade, da raça branca, foi hospitalizada no Serviço de Reanimação Clínica do Hospital Civil de Estrasburgo, em esta-

\footnotetext{
Trabalho realizado no Departamento de Cirurgia da Faculdade de Ciências Médicas da Universidade Estadual de Campinas. Campinas. SP. Brasil e no Service de Réanimation Médicale de I'Université Louis Pasteur. Strasbourg, France.

Recebido para publicaçăo em 2 de dezembro de 1988.

- Do Departamento de Cirurgia da Faculdade de Ciências Médicas da Universidade de Campinas.

* Do Service de Réanimation Médicale, Université de Strasbourg. France.

Endereço para separatas: Anibal Basile Filho. Av. José Bonifácio, 1025, Bloco 5, Apto. 11. 13093, Campinas, SP. Brasil
} 
BASILE FILHO, A.; JAEGER, A.; CAPONE NETO, A.; MANTOVANI, M. - Superinfecção e rotura de aneurisma da aorta abdominal por Salmonella dublin: relato de caso. Rev. Bras. Cir. Cardiovasc., 3(3): 220-223, 1988.

do de choque. A notar, em seus antecedentes, 2 anos antes, queixa de palpitação e precordialgia, e diagnóstico de aneurisma da aorta abdominal estabelecido por aortografia.

Algumas horas antes da admissão, a paciente referiu cefaléia, vertigens, calafrios, dor abdominal na região epigástrica e na base do hemitórax direito, de forte intensidade. Ao exame, apresentava confusão mental, calafrios, pele fria, sudorese de extremidades, taquipnéia e cianose generalizada. A temperatura era de $39^{\circ} \mathrm{C}$, o pulso era de $140 \mathrm{bpm}$ e a pressão arterial, de 70×30 $\mathrm{mmHg}$. A ausculta do tórax revelava diminuição do murmúrio vesicular de todo o hemitórax direito. As bulhas cardiacas eram rítmicas, hiperfonéticas, não se evidenciando sopros. Os pulsos estavam presentes, bilaterais, porém fracos. A palpação abdominal evidenciou massa pulsátil na região epigástrica de, aproximadamente, 15 $\mathrm{cm}$ de diâmetro. A ausculta do abdômen era normal. No plano bioquímico notava-se: uréia $=189 \mathrm{~g} / \mathrm{l}$, creatinina $=2.5 \mathrm{mg} \%$, hematócrito $=34 \%$, hemoglobina $=10.4 \mathrm{~g} / \mathrm{l}$, leucócitos $=10500 / \mathrm{mm}^{3}$ com desvio acentuado à esquerda. A gasometria arterial mostrava $\mathrm{pH}$ $=7,30, \mathrm{PaO} 2=50$ Torr, $\mathrm{PaCO} 2=25$ Torr e bicarbonato $=17 \mathrm{mEq} / \mathrm{l}$. Na hemocultura realizada, durante a admissão, houve crescimento de bacilos gram negativos identificados, a seguir, como Salmonella dublin. O estudo radiológico do tórax mostrou derrame pleural importante à direita e um infiltrado pulmonar alvéolo-intersticial basal direito, além de sinais de calcificação da parede da croça da aorta e ausência de cardiomegalia. A radiografia simples do abdômen foi considerada normal.

O tratamento inicial constou de drenagem do hemitórax direito, com retirada de $2000 \mathrm{ml}$ de liqüido sero-sangüinolento, em cuja cultura isolou-se Salmonella dublin. Instituiu-se antibioticoterapia com ampicilina ( $8 \mathrm{~g} / \mathrm{dia})$, e a paciente referiu discreta melhora da dispnéia. No entanto, algumas horas depois, houve degradação dos sinais vitais, com piora do quadro infeccioso e piora da dor abdominal. Realizou-se ultra-sonografia abdominal, à beira do leito, que mostrou volumoso aneurisma da aorta abdominal de $7 \mathrm{~cm}$ de diâmetro e se observou um ematoma peri-aneurismático, ocupando todo o espaço retroperitoneal (Figura 1). Em virtude da queda do hematócrito e da hemoglobina, a paciente foi transferida para o Serviço de Cirurgia Cardiovascular, onde foi submetida a cirurgia. Os achados cirúrgicos incluíram presença de um grande aneurisma da aorta abdominal de $10 \times 10 \mathrm{~cm}$, logo abaixo do diafragma. Havia, em toda a extensāo de sua parede, microabcessos. O restante da aorta abdominal mostrava-se endurecida, irregular, com grandes áreas de calcificação. $\mathrm{Na}$ abertura de sua parede, evidenciaram-se placas de ateroma e, na porção lateral direita, havia uma rotura intimal, parcialmente tamponada, de $1 \mathrm{~cm}$, que se extendia diagonalmente através da túnica muscular, até a adventícia, $5 \mathrm{~cm}$ abaixo. Ao lado da adventícia, notou-se um enorme trombo e a presença de $2000 \mathrm{ml}$ de sangue no retroperitônio. Obser- vou-se, ainda, uma coloração azulada nas alças do delgado, sugerindo um início de comprometimento isquêmico do intestino. Após o pinçamento da aorta abdominal, $3 \mathrm{~cm}$ do aneurisma, este foi ressecado, colocando-se, em seu lugar, uma prótese de Dacron. A paciente manteve-se em choque, durante todo o ato cirúrgico. vindo a falecer no pós-operatório imediato. Isolou-se. na cultura da parede do aneurisma, Salmonella dublin.

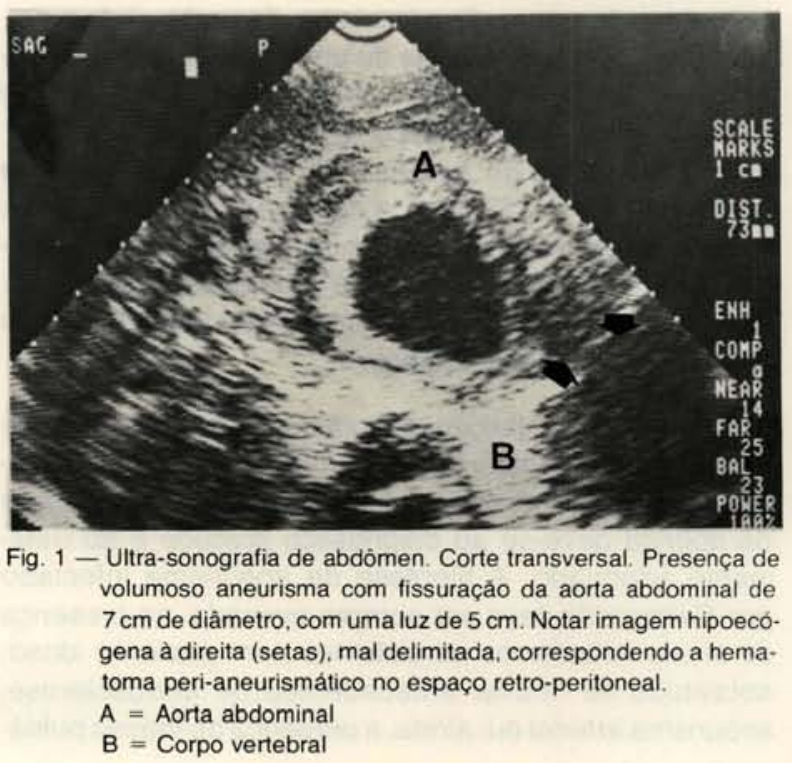

\section{COMENTÁRIOS}

A salmonelose pode infectar qualquer tecido orgânico, durante ou após uma bacteremia. A porta de entrada da Salmonella é, provavelmente, intestinal. Porém, apenas $30 \%$ dos pacientes apresentam bacteremia durante uma gastroenterite ${ }^{3}$. Embora se conheça o fato de a salmonelose causar infecçōes metastáticas, as formas extra-intestinais são raras. As manifestaçōes focais, estimadas em $10 \%$ das septicemias ${ }^{14}$, compreendem focos à distância da porta de entrada. Assim, pode-se observar, durante uma infecção por Salmonella, meningite, pneumonia, osteoartrite, espondilodiscite e infecções da esfera cardiovascular, como endocardite, pericardite e complicaçōes arteriais.

As bacteremias causadas por Salmonella non-typhi são devidas, em mais de $90 \%$ dos casos, à $S$. choleraesuis, $S$. typhi-murium e, mais raramente, $S$. dublin ${ }^{6}$. A freqüência de infecções cardiovasculares, sobretudo as arteriais, parece ser mais elevada nos pacientes com idade superior a 50 anos e deve estar ligada ao aumento da incidência de aterosclerose, a partir dessa faixa etária $^{12,17,19,20}$. COHEN et alii ${ }^{4}$ demonstraram que $25 \%$ dos pacientes, com mais de 50 anos, desenvolveram infecção endovascular, durante bacteremia por Salmonella. Estes mesmos autores observaram, ao revisar a literatura, que, dos 46 casos descritos de pacientes com 
BASILE FILHO, A.; JAEGER, A.; CAPONE NETO, A.; MANTOVANI, M. - Superinfecção e rotura de aneurisma da aorta abdominal por Salmonella dublin: relato de caso. Rev. Bras. Cir. Cardiovasc., 3(3): 220-223, 1988.

lesão arterial por Salmonella, apenas 3 apresentavam idade inferior a 50 anos.

As complicaçōes arteriais constituem-se na formação de aneurismas, ditos micóticos, após disseminação hematogênica, ou pela superinfecção de aneurismas pré-existentes, como no caso relatado. Contudo, a Salmonella dublin está raramente implicada nas complicações arteriais. MILLER ${ }^{13}$, em 1954, havia relatado um caso fatal de rotura de aneurisma da aorta abdominal, infectado, por contigüidade de um processo de espondilodiscite. LAURENT et alii ${ }^{9}$ observaram a formação de aneurisma filio-femoral, após septicemia por $S$. dublin. A superinfecçāo de aneurismas pré-existentes pode ocorrer de quatro maneiras: a agressão direta sobre uma fragilidade prévia do endotélio vascular, durante disseminação hematogênica; através de vasos linfáticos; através dos vasa-vasorum, ou por contigüidade de um processo infeccioso peri-aneurismático ${ }^{5}$.

Até 1962, a infecção da aorta abdominal era sempre fatal ${ }^{18}$. Contudo, até o final de 1985 , registrou-se a sobrevivência de 12 pacientes. Essa modificaçāo no curso da doença deve-se ao diagnóstico precoce e ao tratamento adequado. A hipótese de aneurisma infectado por Salmonella deve ser sempre evocado, na presença de sinais infecciosos persistentes num paciente idoso, sobretudo se houver antecedentes de aterosclerose, aneurisma arterial ou, ainda, a presença de massa pulsá- til na linha média do abdômen. Nesse caso, o procedimento diagnóstico mais recomendado é a ultra-sonografia abdominal, exame que pode ser facilmente realizado à beira do leito. As informaçōes obtidas sâo rápidas e valiosas, podendo mudar a abordagem ao paciente ${ }^{\text {. }}$ 11. É claro que o diagnóstico precoce favorece a estratégia clínica e cirúrgica a ser adotada. A prótese de Dacron utilizada após a ressecção do aneurisma freqüentemente se infecta ${ }^{19}$. Assim, a cirurgia que se tornou mais popularizada é a que emprega o enxerto axilo-bi-femoral, acompanhado de ressecção do aneurisma ${ }^{15}{ }^{16}$. Este procedimento, descrito, simultaneamente, por BLAISDELL \& HALL ${ }^{2}$ e LOUW $^{10}$, em 1963, mereceu destaque. pois propicia um excelente tempo de sobrevida aos pacientes.

Ao lado da cirurgia, a antibioticoterapia é outro ponto crítico. No tratamento da superinfecção de aneurismas por Salmonella, a combinação cloranfenicol e ampicilina parece ser a mais eficaz, na opinião de vários autores ${ }^{8}$ 17. 18 . No caso de alergia às lactaminas, a associação trimetropim/sulfametoxazol tem sido aceita, como via alternativa no tratamento da salmonelose ${ }^{7}$.

Enfim, se o diagnóstico de superinfecçāo do aneurisma por Salmonella for precoce, acredita-se que a abordagem clínico-cirúrgica descrita pode modificar o curso fatal apresentado pelos pacientes.

\section{RBCCV 44205-71}

BASILE FILHO, A.; JAEGER, A.; CAPONE NETO, A.; MANTOVANI, M. - Superinfection and rupture of abdominal aortic aneurysm after Salmonella dublin septicemia: a case report. Rev. Bras. Cir. Cardiovasc., 3(3): 220-223, 1988.

ABSTRACT: The authors present a case of a 66 year-old woman with a dissection and rupture of abdominal aortic aneurysm after Salmonella dublin septicemia. Endovascular infection and rupture of atherosclerotic aneurysm is a substantial risk in patients older than 50 years of age who have bacteremia or sepsis due to Salmonella sp. The high mortality is the resultant of Salmonella aortitis due either to septicemia or rupture. Tissue anywhere may be seeded, but damage tissues appear to be particularly susceptible to abscess and chronic destructive lesions. The significance of early diagnosis to decrease the fatal outcome is discussed. surgery.

DESCRIPTORS: abdominal aortic aneurysm, rupture, Salmonella dublin; abdominal aortic aneurysms,

\section{REFERÊNCIAS BIBLIOGRÁFICAS}

1 BASILE FILHO, A. \& NANI, L. - A imaginologia do paciente crítico. Rev. Terap. Intensiva, 2(4): 97-104, 1988.

2 BLAISDELL, F. W. \& HALL, A. D. - Axillary-femoral artery bypass for lower extremity ischemia. Surgery, 54: 563-568, 1963.
3 CHERUBin, C. E.; NEU, H. C.; IMPERATO, P. J.; HARVEY, R. P.;BELLEN, N. - Septicemia with non typhoid salmonella. Medicine (Baltimore) 53: 365-376, 1974. MEDEIROS, A. A. - The risk of endothelial infection in adults with salmonella bacteremia. Ann. Inter. Med., 89: 931-932, 1978 
BASILE FILHO, A.; JAEGER, A.; CAPONE NETO, A.; MANTOVANI, M. - Superinfecção e rotura de aneurisma da aorta abdominal por Salmonella dublin: relato de caso. Rev. Bras. Cir. Cardiovasc., 3(3): 220-223, 1988.

5 DEVOLFE, C.; BARRAL, X.; LESCOEUR, N.; NINET, J. DESCOTES, J.; PASQUIER, J. - Anéurysme infectieux de l'aorte abdominale et septicémie à salmonella: évolution favorable à 2 ands d'un cas traité par resection et pontage axillo-bi-fémoral. J. Mal. Vasc., 7: 339-344, 1982.

6 FOOTE, S. C. \& HOOK, E. W. - Salmonella species. In: MANDELL, G. L.; DOUGLAS, R. G.; BENNET, J. E. eds. Principles and practice of infectious diseases. New York, Wiley, 1979. p. 1730-1750.

7 GILMAN, R. H.; TERMINEL, M.; LEVINE, M. M.; HERNANDEZ-MENDONSA, P.; CALDERONE, E. - Comparison of trimethoprim-sulphamethoxazole and amoxicillin in therapy of chloramphenicol-resistant and chloramphenicol-sensitive typhoid fever. J. Infect. Dis., 132: 630-636, 1975.

8 HOOK, E. W. \& GUERRANT, R. L. - Therapy of infective endocarditis. In: KAYE, D. ed. Infective endocarditis. Baltimore, University Park Press, 1976. p. 167-169.

9 LAURENT, J. M.; DUCLOUX, G.; DELMOTTE, C.; RAPAPORT, C.; COPROS, M.; WAREMBOURG Jr., J. Anévrysme artériel à Salmonella dublin: a propos d'un cas opéré. Larc. Méd., 5: 395-397, 1982.

10 LOUW, J. H. - Splenic-to-femoral and axillary-to-femoral bypass grafts in diffuse atherosclerotic occlusive disease. Lancet, 1: 1401-1402, 1963.

11 MEADE, R. H. \& MORAN, J. M. - Salmonella arteritis: preoperative diagnosis and cure of Salmonella typhimurium aortic aneurysm. N. Engl. J. Med., 281: 310-312, 1969.
12 MENDELOWITZ, D. S.; RAMSTEDT, R.; YAO, J. S. T. BERGAN, J. J. - Abdominal aortic salmonellosis. Surgery, 85: 514-519, 1979

13 MILLER, A. A. - Salmonella dublin osteomyelitis of the spine: report of a fatal case. Brit. Med. J., 4: 194-195, 1954.

14 MOREILLON, P.; FRANCIOLI, P.; DELACRETAZ, F.; WEBER, J.; GLAUSER, M. P. - Les infections endo-artérielles, complications rédoutables des infections à salmonella non typhi. Schweiz. Med. Wschr., 113: 1953-1955, 1983.

15 PARSONS, R.; GREGORY, J.; PALMER, D. L. - Salmonella infections of the abdominal aorta. Rev. Infect. Dis., 5(2): 227-231, 1983.

16 PORSCHEN, R. K.; HALE, D.; GOODMAN, Z. - Misdiagnosed salmonella septicemia and endarteritis due to a lactose-fermenting strain. $A m$. J. Surg., 136: 96-101, 1978.

17 REICHLE, F. A.; TYSON, R. R.; SOLOFF, L. A.; LAUTSCH, E. V.; ROSEMOND, G. P. - Salmonellosis and aneurysm of the distal abdominal aorta: case report with a review. Ann. Surg., 171: 219-228, 1970.

18 SOWER, N. D. \& WHELAN Jr., T. J. - Suppurative arteritis due to Salmonella. Surgery, 52: 851-859, 1962.

19 WILSON, S. E.; GORDON, H. E.; VAN VAGENEN, P. B. - Salmonella arteritis. Arch. Surg., 113: 1163-1166, 1978.

20 ZAK, F. G.; STRAUSS, L.; SAPHRA, I. - Rupture of diseased large arteries in the course of enterobacterial (salmonella) infections. N. Engl. J. Med., 258: 824-828, 1958. 